MS37-P02

\section{(Mechano)synthesis, structure characterization and pharmacological evaluation of harmine derivatives as new anticancer compounds}

\author{
Laurie Bodart ${ }^{1}$, Sébastien Marx ${ }^{1}$, Céline Meinguet ${ }^{1}$, Nikolay \\ Tumanov $^{1}$, Johan Wouters ${ }^{1}$ \\ 1. Namur Medicine \& Drug Innovation Center (NAMEDIC- \\ NARILIS), Laboratoire de Chimie Biologique Structurale (CBS), \\ University of Namur, Namur, Belgium
}

email: laurie.bodart@unamur.be

Harmine is a natural $\beta$-carboline compound, which can be isolated from Peganum Harmala, presenting antiproliferative activity. Trisubstituted harmine derivatives were shown to be more potent than mono- and di-substituted ones [1, 2]. Here, new trisubstituted harmine derivatives were designed and synthetized in our group with the aim to overcome the intrinsic resistance of cancer cells to apoptotic stimuli.

The last step of synthesis is performed by mechanosynthesis and compounds involved in this synthesis are characterized by single-crystal X-ray diffraction. However, trisubstituted harmine derivatives often present a moderate solubility at $\mathrm{pH}$ 7.4. In this context cyclodextrin complexes were already prepared in our group to improve compounds solubility [2]. Here we propose a new approach to reach this objective. Indeed, the third substituent chosen to be placed on the harmine core contains a pyridine moiety in order to improve compound solubility in comparison with benzyl moiety. Moreover, pyridine increases the tendency towards cocrystal/salt formation. This is also considered as a potential method to modulate the solubility of the compound [3]. The aim of this work is to obtain novel druggable harminebased molecules combining antiproliferative activity in micromolar to submicromolar range and a high solubility at physiological $\mathrm{pH}$ in order to allow intravenous injection of these compounds.

L.B. thanks the Fonds National pour la Recherche Scientifique-FNRS for her grant and the PC2 technological platform of UNamur.

References:

[1] Meinguet, C. et al. (2015). Eur. J. Med. Chem. 94, 45-55.

[2] Meinguet, C. et al. (2015) Eur. J. Pharm. Sci. 77, 135-140.

[3] Pharmaceutical Salts and Co-crystals, (2012). Edited by J.

Wouters \& L. Quéré, pp. 116-119. RSC Publishing.

Keywords: Harmine, mechanosynthesis, crystal engineering
MS37-P03

\section{Study of thermo- and photochromic behaviour of a hydrazone system obtained by mechanosynthesis}

Mégane Van Gysel ${ }^{1}{ }^{2}$, Andrea Carletta ${ }^{1}$, Nikolay Tumanov ${ }^{1}$, Johan Wouters ${ }^{1}$

1, Namur Medicine \& Drug Innovation Center (NAMEDICNARILIS), Laboratoire de Chimie Biologique Structurale (CBS), University of Namur, Namur, Belgium

2. Namur Institute of Structured Matter (NISM), Laboratoire de Chimie Biologique Structurale (CBS), University of Namur, Namur, Belgium

email: megane.vangysel@unamur.be

Hydrazones are compounds widely studied. They can be made by several synthetic pathways, such as a condensation reaction between a hydrazine or hydrazide with a ketone or aldehyde [1]. These molecules are constituted by an azomethine group, characterized by the fragment $\mathrm{C}=\mathrm{N}-\mathrm{N}$, allowing the use of hydrazones in various fields of chemistry. These molecules have many potential applications: they are used in dyes and in conductive materials to name a few [1]. In pharmaceutical field, several hydrazone-based prodrugs of isoniazid have shown less undesired side effects when compared with isoniazid [2]. These molecules can also be studied for their potential thermo- and photochromic properties.

Thermo- and photochromism are color change phenomena induced by temperature variation and absorption of electromagnetic radiations, respectively. These phenomena have many applications: information storage, optical switching devices (optical glasses), etc. [3].

In this work, we are interested in a hydrazone system obtained by a condensation reaction between isoniazid and $o$-vanillin. This molecule is cocrystallized with various cocrystal formers with the aim to modulate its photo- and thermochromism [3]. Both syntheses of the chromophore and of its cocrystals are accomplished by mechanosynthesis. All obtained solid forms are characterized by powder X-ray diffraction and single-crystal X-ray diffraction. Photochromism is studied by UV-Vis diffuse reflectance.

Authors acknowledge ARC (UNamur - UCL) for financial support.

References:

[1] Su, X. \& Aprahamian, I. (2014). Chem. Soc. Rev., 43, 19631981.

[2] Ferraresi-Curotto, V. et al. (2017). J. Mol. Struct., 1133, 436-447.

[3] Carletta, A. et al. (2017). Chem. Eur. J., 23, 5317-5329.

Keywords: mechanosynthesis, cocrystals, hydrazones 